
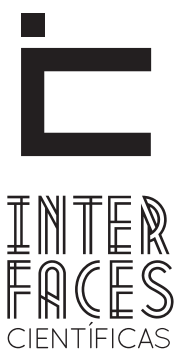

SAÚDE E AMBIENTE

\title{
QUALIDADE DE VIDA NO TRABALHO DOS PROFISSIONAIS DE ENFERMAGEM: UMA ANÁLISE DA PRODUÇ̃̃O CIENTÍFICA ENTRE 2O00 E 2012
}

Carla Maria Couto Silveira ${ }^{1}$

Ilva Santana Santos Fonseca ${ }^{3}$
Rosiane Barbosa da Silva²

Maria da Pureza Ramos de SantaRosa ${ }^{4}$

\section{RESUMO}

A qualidade de vida não está atrelada apenas a um aspecto da vida do homem, ela está associada a diversos fatores que determinam que o indivíduo possa estar em harmonia bio-psico-socio-cultural. Ter qualidade de vida depende, pois de fatores intrínsecos e extrínsecos. Ao abordarmos qualidade de vida no trabalho esta é fundamental em qualquer atividade, especialmente na enfermagem, que tem um enfoque direcionado para melhoria da qualidade de vida da população. Esse estudo objetivou fazer um levantamento bibliográfico e análise das produções científicas acerca do tema Qualidade de Vida (QV) e Qualidade de Vida no Trabalho (QVT), publicadas nos principais periódicos brasileiros na área de Enfermagem, no perío- do de 2000 a 2012. Os resultados apresentados nesse estudo indicam que os construtos de QV e QVT têm conotações singulares e muito importantes a serem exploradas e aprofundadas, especialmente em relação ao trabalhador de enfermagem, pois sua atuação é indispensável na área da saúde. Foi observado que o tema qualidade de vida no trabalho do profissional de enfermagem é um tema recente e pouco abordado no meio científico.

\section{PALAVRAS-CHAVE}

Qualidade de Vida. Qualidade de Vida no Trabalho. Enfermagem. 


\section{ABSTRACT}

The quality of life is linked not only to one aspect of human life; it is associated with many factors that determine the individual may be bio-psycho-socio-cultural harmony. Have quality of life depends on intrinsic and extrinsic factors. In addressing quality of work life, this is critical in any activity, especially in nursing who has a targeted approach to improving the quality of life. This study aimed to review the literature and analysis of scientific production about Quality of Life and Quality of Work Life, published in major Brazilian journals in Nursing in the period 2000-2012. The re- sults presented in this study indicate that the constructs of QOL and QWL have natural connotations and very important to be explored and deepened, especially in relation to nursing workers, because its activity is essential for health. It was observed that the subject quality of work life of nursing staff is a recent topic and little attention in the scientific community.

\section{KEYWORDS}

Quality of Life. Quality of Work Life. Nursing.

\section{RESUMEN}

La calidad de vida no se resume a un solo aspecto de la vida del hombre, puesto que la misma está asociada a diversos factores que determinan que el individuo pueda estar en harmonía bio-psico-socio-cultural a su entorno. Tener calidad de vida, se supone depender de factores intrínsecos y extrínsecos. Al abordar la calidad de vida en el trabajo, esto es esencial en cualquier actividad, sobre todo en enfermería que tiene un enfoque dirigido a la mejora de la calidad de vida de la populación. Este estudio tuvo como objetivo revisar la literatura y el análisis de las publicaciones científicas sobre el tema de Calidad de Vida y Calidad de Vida Laboral, publicado en las principales revistas brasileñas en el campo de la enfermería, en el período 2000-2012. Los resultados presentados en este estudio, indican que construcciones de la CDV y QWL son de singular importancia para ser explorados en profundidad, sobre todo en lo que dice respecto a los trabajadores de enfermería, ya que su actuación es esencial en la asistencia sanitaria. Se observó que el tema de la calidad en la vida laboral del personal de enfermería es un tema reciente y poco abordado en la comunidad científica.

\section{PALABRAS CLAVE}

Calidad de vida. Calidad de vida laboral. De enfermería. 


\section{INTRODUCÃO}

Qualidade de vida (QV) é uma expressão de difícil conceituação, tendo em vista o seu caráter subjetivo, complexo e multidimensional. Ter qualidade de vida depende, pois, de fatores intrínsecos e extrínsecos. Assim, há uma conotação diferente de qualidade de vida para cada indivíduo, que é decorrente da inserção desse na sociedade (ROCHA; FELLI, 2004).

A QV não está atrelada apenas a um aspecto da vida do homem, ela está associada a diversos fatores que determinam que o indivíduo possa estar em harmonia bio-psico-socio-cultural. Isso envolve saúde física, saúde mental, relações familiares, relações interpessoais, satisfação no trabalho, entre outras (KANAANE, 1999 apud SIQUEIRA JUNIOR et al., 2006).

Segundo a Organização Mundial de Saúde (OMS) o termo qualidade de vida é definido como: "A percepção do indivíduo de sua posição na vida no contexto da cultura e sistema de valores nos quais ele vive e em relação aos seus objetivos, expectativas, padrões e preocupações" (FLECK, 1999 apud NEVES et al., 2010).

A expressão QV possui vários conceitos diferentes de acordo com cada sociedade e cultura, cada uma possui sua percepção em relação à qualidade de vida (ZHAN, 1992 apud KAWAKAME, MIYADAHIRA, 2005).

Após a segunda guerra mundial, nos Estados Unidos a qualidade de vida era sinônimo de bens materiais como ter dinheiro, imóveis, automóveis, investimentos, viagens, entre outros. Posteriormente, o conceito foi ampliado com o objetivo de se medir o desenvolvimento social e econômico e de uma determinada sociedade por meio de saúde, educação, transporte, moradia entre outros (ZHAN, 1992 apud KAWAKAME, MIYADAHIRA, 2005).
Atualmente, o tema qualidade de vida tem sido muito discutido através dos meios de comunicação e está constantemente no discurso popular, tornando-se bastante difundido e sendo alvo de pesquisa de várias áreas do conhecimento (FARQUHAR, 1995 apud KAWAKAME; MIYADAHIRA, 2005).

A QV não depende somente de fatores relacionados à saúde, mas envolve trabalho, família, amigos e outras circunstâncias da vida, e é fundamental para a execução de qualquer atividade inclusive na enfermagem, que tem um enfoque direcionado para a melhoria da qualidade de vida da população, tornando-se primordial que o próprio trabalhador de enfermagem adquira plenas condições de trabalho e de vida (SIQUEIRA JÚNIOR, 2006).

A Qualidade de Vida no Trabalho (QVT) é outro tema amplamente discutido, já que o indivíduo passa a maior parte do tempo no trabalho. Dessa maneira, entende-se como QVT o conjunto de características do trabalhador e aspectos situacionais do contexto do trabalho (CHIAVENATO, 1999 apud FARIAS; ZEITOUNE, 2007).

A necessidade de funcionamento diuturno, que implica na existência de regime de turnos e plantões, permite a ocorrência de duplos empregos e longas jornadas de trabalho, comuns entre os trabalhadores da saúde, especialmente quando os salários são insuficientes para a manutenção de uma vida digna. Tal prática compromete a Qualidade de Vida bem como a QVT (PITTA, 1991 apud ELIAS, NAVARRO, 2006).

No Brasil, os profissionais de enfermagem têm reconhecidamente longas jornadas de trabalho. Os plantões de 12 horas seguidos por 36 ou 60 horas de descanso permitem que esses profissionais se dediquem a mais de uma atividade produtiva. Nesse grupo profissional, as longas jornadas podem levar à exaustão e fadiga, podendo afetar a assistência aos pacientes. Além disso, em função da predomi- 
nância feminina, a jornada de trabalho profissional se adiciona ao trabalho doméstico, compondo a chamada jornada total ou carga total de trabalho (SILVA et al., 2011).

Diante do exposto o presente trabalho teve como objetivo verificar a produção científica sobre qualidade de vida no trabalho dos profissionais de enfermagem no Brasil entre 2000 e 2012.

\section{METODOLOGIA}

A presente pesquisa se trata de uma revisão bibliográfica centralizada no levantamento, descrição e análise de produções científicas publicadas nos principais periódicos da área de enfermagem que abordam a temática de Qualidade de Vida no Trabatho dos profissionais de enfermagem, nos anos de 2000 a 2012.

Foram realizadas pesquisas na base de dados da Biblioteca Virtual de Saúde (BVS) - Enfermagem, um espaço virtual da Internet formado por informações primárias e secundárias em enfermagem produzidas no Brasil, que compõem os recursos ou fontes de informação onde usuários de diferentes níveis e espaços poderão utilizar essas informações independentemente de sua localização física.

Para seleção dos artigos foram utilizados os seguintes descritores: qualidade de vida, qualidade de vida no trabalho, processo de trabalho de enfermagem. Como critérios de inclusão, foram considerados: texto completo disponível; textos publicados em base de dados no Brasil; ano de publicação (2000 a 2012), idioma (português), tipo de publicação (artigo), país (Brasil).

Foi estabelecido apenas o idioma português na publicação, a fim de se verificar a produção em território nacional, por parte da Enfermagem brasileira.
Ainda como critério de inclusão para os artigos analisados, estes deveriam ter sido publicados em periódicos indexados e deter conceituação avaliativa entre $\mathrm{A} 1$ a B5, conforme critérios definidos pela fundação Coordenação de Aperfeiçoamento de Profissional de Ensino Superior (CAPES), do Ministério de Educação. Os artigos com conceito $C$ foram desconsiderados, por representar peso zero para a CAPES.

Para a coleta dos dados bibliográficos, foi elaborado e utilizado um instrumento contendo as seguintes informações: ano de publicação, periódico, classificação Capes, título, autores, tipo de estudo, palavras-chave, área de conhecimento, objetivo do estudo, resultados obtidos e conclusão.

A análise dos dados foi realizada por meio da descrição de estudos e informações, análise dos resultados dos artigos, compilação destes em assuntos principais, como: conceito de QV e QVT, QV e a formação acadêmica do estudante de enfermagem, qualidade de vida e profissão enfermagem e avaliação dos resultados sobre a temática proposta.

\section{RESULTADOS E DISCUSSÕES}

Ao se buscar os artigos na BVS - Enfermagem, utilizando-se os critérios de inclusão definidos na pesquisa, como texto completo disponível; textos publicados em base de dados nacional; ano de publicação (2000 a 2012), idioma (português), tipo de publicação (artigo), país (Brasil), foram identificados, a principio, 123 artigos, distribuídos em 23 periódicos.

Após se inserir o critério de inclusão classificação qualiCapes e utilizar os descritores estabelecidos de forma associada, foram encontrados 27 artigos em 9 periódicos. Destes 27, apenas 19 tratavam diretamente da temática qualidade de vida no trabalho e profissionais de enfermagem (TABELA 1). 
Tabela 1 - Periódicos utilizados no estudo segundo critérios de avaliação da CAPES, 2012

\section{Periodicos da área de enfermagem}

Avaliação da capes

Revista Latino-Americana de Enfermagem (USP. Ribeirão Preto. Impresso)

Cadernos de Saúde Pública (ENSP. Impresso)

Revista da Escola de Enfermagem da USP (Impresso)

A2

Arquivos de Ciências da Saúde (FAMERP)

REME. Revista Mineira de Enfermagem

Escola Anna Nery

Revista Eletrônica de Enfermagem

Fonte: Biblioteca Virtual de Saúde - Enfermagem, Bireme / OPAS / OMS, MS. 2013.

Entre os periódicos com maior número de publicação, destacam-se a Revista Latino-Americana de Enfermagem, com 31,6\% das produções, a Revista da
Escola de Enfermagem da USP com $21,1 \%$ e os periódicos Arquivos de Ciências da Saúde e Cadernos de Saúde Pública, com 10,5\%, ambos (TABELA 2).

Tabela 2 - Distribuição dos periódicos utilizados no estudo

\begin{tabular}{lrr}
\hline Periódicos & \multicolumn{2}{c}{ Quantitativo } \\
\hline & $\mathrm{n}$ & $\%$ \\
Revista Latino-Americana de Enfermagem (USP. Ribeirão Preto. Impresso) & 6 & 31,6 \\
Cadernos de Saúde Pública (ENSP. Impresso) & 2 & 10,5 \\
Revista da Escola de Enfermagem da USP (Impresso) & 4 & 21,1 \\
Arquivos de Ciências da Saúde (FAMERP) & 2 & 10,5 \\
REME. Revista Mineira de Enfermagem & 1 & 5,3 \\
Escola Anna Nery & 1 & 5,3 \\
Revista Eletrônica de Enfermagem & 1 & 5,3 \\
Revista do Instituto de Ciências da Saúde (UNIP) & 1 & 5,3 \\
Revista Enfermagem UERJ & 1 & 5,3 \\
TOTAL: & 19 & 100 \\
\hline
\end{tabular}

Fonte: Biblioteca Virtual de Saúde - Enfermagem, Bireme / OPAS / OMS, MS. 2013.

Em relação ao quantitativo de publicações anuais, verificou-se uma similaridade/estabilidade ao longo do período que abrange a presente investigação, sendo que de 2000 a 2002 não foi encontrado nenhum artigo publicado e aos demais anos observou-se um percentual que varia em torno de 5 a $15 \%$ das publicações analisadas. Esse dado sugere que a QVT dos profissionais de enfermagem é um assunto recente na discussão acadêmica, dentro da área de enfermagem conforme demonstrado na tabela 3. 
Tabela 3 - Distribuição dos artigos catalogados entre 2000 a 2012, na BVS - Enfermagem de acordo com os critérios de avaliação da CAPES

\begin{tabular}{lrr}
\hline Ano & $n$ & $\%$ \\
\hline 2000 & 0 & 0,0 \\
2001 & 0 & 0,0 \\
2002 & 0 & 0,0 \\
2003 & 1 & 5,3 \\
2004 & 2 & 10,5 \\
2005 & 2 & 10,5 \\
2006 & 3 & 15,8 \\
2007 & 1 & 5,3 \\
2008 & 3 & 15,8 \\
2009 & 3 & 15,8 \\
2010 & 2 & 10,5 \\
2011 & 1 & 5,3 \\
2012 & 1 & 5,3 \\
Total & 19 & 100,0 \\
\hline
\end{tabular}

Fonte: Biblioteca Virtual de Saúde - Enfermagem, Bireme / OPAS / OMS, MS. 2013.

Quanto ao conceito inicial Qualidade de vida (QV) na produção cientifica analisada, verificou-se que o constructo QV não apresenta uma definição consolidada e unificada. Diversos autores trazem conceitos que abrangem questões subjetivas e que dizem respeito à percepção de cada indivíduo. Definem itens como nível socioeconômico, valores, culturais éticos e religiosos, ausência de doença, lazer e relações interpessoais saudáveis como requisitos para uma boa qualidade de vida.

De acordo com Seid e Zannon (2004), o construto QV apresenta natureza multidimensional, subjetiva e interdisciplinar, fazendo com que várias áreas do conhecimento busquem estudos acerca dessa temática.

Cada indivíduo, em diferentes momentos e circunstâncias da sua vida, pode perceber a qualidade de vida de uma ótica diferente, o que faz com que vários aspectos sejam caracterizados e con- ceituados dentro deste constructo. Tais aspectos vão desde uma vida saudável fisicamente, até a harmonia e equilíbrio entre as esferas psicológicas, espirituais, sociais e econômicas.

A abordagem da QV dentro do ambiente de trabalho é entendida como QVT e este conceito na enfermagem, também, apresenta caráter subjetivo. Kimura e Carandina (2009) ao desenvolver uma versão reduzida de um instrumento para avaliação da QVT dos profissionais de enfermagem observou que esta não se refere apenas ao contexto do trabalho e estrutura organizacional, mas, também, a sentimentos, aspirações, crenças e valores, ratificando que QVT é um construto multidimensional e subjetivo, assim como foi observado em QV.

Durante a academia já se observa o impacto negativo que a profissão causa na QV dos discentes, visto que aspectos relacionados à saúde ficam comprometidos durante a sua vida acadêmica.

Estudos de Souza e outros autores (2012), mostram que durante a graduação já se observa prejuízos na QV relacionada à saúde, pois sintomas depressivos entre os estudantes de enfermagem ao longo do curso só aumentam, principalmente no último ano, uma vez que ao final do curso ressaltam-se as relações conflitantes vividas por estudantes no ambiente hospitalar, justificadas pela visão hegemônica da medicina, que coloca o profissional médico como personagem central e os demais profissionais de saúde como complementares, bem como a reprodução desses conflitos internamente na equipe de enfermagem, com a incorporação do profissional enfermeiro como dominador e as demais categorias como subalternas.

Oliveira e Ciampone (2008) observaram que durante a formação o aluno vivencia no seu processo de aprendizagem tanto os aspectos promotores como os não promotores de QV e defendem que há necessidade de preparo dos futuros profissionais de saúde e dos docentes para o desenvolvimento 
do cuidado de si, como precedente da condição para o cuidar do outro, pois se não há elaboração, tendem a reproduzir o mesmo modelo em suas práticas profissionais.

A profissão de enfermagem em seu cotidiano já traz situações que promovem um desconforto emocional. Para Barros e outros autores (2003) essa profissão é altamente geradora de ansiedade, principalmente por conta de alguns fatores como: relacionamento com a família do paciente, com a equipe de enfermagem e com o serviço de manutenção, falta de suprimentos como, também, falta de pessoal.

Já para Martins e outros autores (2010), a equipe de enfermagem vivencia situações de ansiedade pelo fato de lidar com o sofrimento e a dor dos indivíduos e a convivência diária com a morte, pois ao se deparar com essas situações difíceis de serem superadas, o trabalhador, também, sofre.

Os estudos analisados envolveram basicamente motivação, relacionamento com os colegas, status profissional, remuneração, jornada de trabalho e saúde como indicadores para QVT, tais aspectos interferem entre si, refletindo na satisfação no trabalho.

No que diz respeito à qualidade de vida associada ao relacionamento com os colegas, observou-se entre os artigos lidos que quando não há um bom relacionamento o trabalho tende a ficar mais estressante e desgastante, pois este perde sua característica de trabalho em equipe, uma vez que os indivíduos realizam suas atribuições de maneira individualizada, influenciando, também, diretamente na motivação relacionada ao trabalho.

Para Farias (2007), os profissionais de enfermagem entendem que a integração social na instituição é um determinante para obtenção de Qualidade de Vida no Trabalho naquele ambiente, pois acaba gerando coesão nas ações dentro da equipe, passando então o trabalho a ser desenvolvido de forma integrada.

Outro aspecto, já relacionado à motivação, refere-se a uma remuneração adequada, visto que é por meio do trabalho que o indivíduo procura satisfazer suas aspirações e alcançar sua qualidade de vida. Ao longo do tempo os profissionais de enfermagem vêm buscando salários mais justos e condizentes com a importância da sua profissão, pois o cuidar do outro é algo que deve ser escolhido por vocação, já que é uma prática de difícil execução (ARAÚJO et al., 2009).

A qualidade de vida do profissional pode interferir diretamente na assistência prestada ao paciente, pois entre os estudos encontrados esse é um aspecto que deve ser cuidadosamente analisado, visto que atualmente tem-se observado amplamente a ocorrência de vários erros, muitas vezes fatais, cometidos pela equipe de enfermagem. Isso compromete a imagem e a credibilidade da profissão, principalmente, porque a informação hoje em dia é rapidamente transmitida mundialmente através dos meios de comunicação.

Para Silva e outros autores (2011) no Brasil os profissionais de enfermagem têm longas jornadas de trabalho e o sistema de plantões permite que esses profissionais se dediquem a mais de uma jornada produtiva. Nesse grupo profissional essas longas jornadas podem levar à exaustão e fadiga, podendo comprometer a assistência aos pacientes.

Alguns estudos, também, apontaram o status profissional como condição para uma boa qualidade de vida. O profissional de enfermagem reconhece sua profissão como importante para a sociedade, pois suas atividades estão relacionadas à manutenção da vida, entretanto desde o surgimento da enfermagem, os profissionais buscam um maior reconhecimento. Eles ainda precisam garantir que 
sua atuação é indispensável e merece o mesmo prestígio que outros profissionais da área da saúde. Essa busca de reconhecimento pode comprometer o desempenho de suas atividades e repercutir, também, na sua vida pessoal (SCHMIDT; DANTAS, 2006; SIQUEIRA;KURCGANT, 2014).

Pesquisas revelam que os indivíduos insatisfeitos adoecem mais, sobretudo acometidos por doenças crônicas como depressão e hipertensão. Avendano (apud FARIAS e ZEITOUNE, 2007) diz que na América Latina, pela falta de condições de trabalho, muitas vezes ocorre a deterioração da saúde dos enfermeiros, referindo a profissão como de alto risco.

Para Oler e outros autores (2005), o trabalho é um elemento fundamental para a saúde das pessoas e desde que seja realizado em condições saudáveis, promove sensação de bem-estar, refletindo na melhoria das condições de trabalho, na assistência de enfermagem prestada e, consequentemente, na QV de seus trabalhadores.

Outro fator evidenciado no estudo que está relacionado à saúde do profissional de enfermagem é a carga de trabalho, que tem sido cada vez maior entre os profissionais. Isto se deve ao fato dos vários vínculos que o trabalhador tem, para compensar a baixa remuneração, fazendo com que a jornada de trabalho seja longa, aumentando a carga de trabalho e provocando um desgaste físico e mental (FERREIRA; MARTINO, 2006; MANETTI; MARZIALE;ROBAZZI, 2008; AGUIAR et al., 2009).

Essa sobrecarga pode desencadear prejuízos para a saúde do trabalhador, refletindo na ausência ao trabatho (PIRES et al., 2010). De acordo com Becker e Oliveira (2008), o principal motivo do absenteísmo é por doença.
Isso seria amenizado se fossem elaboradas estratégias de cuidado para a promoção da saúde do trabalhador de enfermagem, a fim de diminuir as ausências da equipe por motivo de doença.

\section{CONSIDERAÇÕES FINAIS}

Os resultados apresentados nesse estudo indicam que os construtos de QV e QVT têm conotações singulares e muito importantes a serem exploradas e aprofundadas, especialmente em relação ao trabalhador de enfermagem, pois sua atuação é indispensável na área da saúde.

Foi observado que o tema qualidade de vida no trabalho do profissional de enfermagem é um tema recente e pouco abordado no meio científico. Podemos ressaltar que apesar da qualidade de vida ter conotação subjetiva, o presente estudo permitiu identificar que aspectos como satisfação, status profissional, relação interpessoal, remuneração e motivação são apontados como indicadores de QVT.

Foi observado ainda que o stress, doenças crônicas, riscos ocupacionais e carga de trabalho excessiva são fatores que interferem consideravelmente na vida social e laboral desses profissionais, podendo trazer riscos à assistência prestada.

Assim, a partir do momento em que o profissional de enfermagem for visto como um indivíduo com direito a um ambiente onde existam formas de trabalho mais favoráveis, este já terá melhores condições em sua QVT, refletindo dessa maneira na sua QV, pois o estresse físico e mental dará lugar ao bem-estar geral. Para tanto é necessário planejamento de programas de promoção à saúde e ações nos ambientes de trabalho para que as condições de trabalho sejam continuamente melhoradas. 


\section{REFERÊNCIAS}

AGUIAR, Ayla Dayane de Faria et al. Saúde do trabalhador de enfermagem que atua em centro de saúde. Rev. Inst. Ciência Saúde. São Paulo, v.27, n.2, p.103-108, 2009.

ARAÚJO, Gisélia Alves; SOARES, Maria Júlia Guimarães Oliveira; HENRIQUES, Maria Emília Romero de Miranda. Qualidade de vida: percepção de enfermeiros numa abordagem qualitativa. Rev. Eletr. Enf. [Internet], v.11, n.3, p.635-41, 2009.

BARROS, Alba Lucia Botura Leite de; HUMEREZ, Dorisdaia Carvalho de; FAKIH, Flávio Trevisaniand MICHEL, Jeanne Liliane Marlene. Situações geradoras de ansiedade e estratégias para seu controle entre enfermeiras: estudo preliminar. Rev. Latino-Am. Enfermagem [online], v.11, n.5, p.585-592, 2003.

BECKER, Sandra Greice; OLIVEIRA, Maria Luiza Carvalho de. Estudo do absenteísmo dos profissionais de enfermagem de um centro psiquiátrico em Manaus, Brasil. Rev. Latino-Am. Enfermagem [online]. v.16, n.1, p. 109-114, 2008.

ELIAS, Marisa Aparecida; NAVARRO, Vera Lúcia. A relação entre o trabalho, a saúde e as condições de vida: negatividade e positividade no trabalho das profissionais de enfermagem de um hospital escola. Rev. Latino-Am. Enfermagem [online], v.14, n.4, p. 517-525, 2006.

FARIAS, Sheila Nascimento Pereira de, ZEITOUNE, Regina Célia Gollner. A Qualidade de Vida no Trabalho de Enfermagem. Esc. Anna Nery [online], v.11, n.3, p. 487-493, 2007.

FERREIRA, Luciane Ruiz Carmona, MARTINO, Milva Maria Figueiredo de. 0 estresse do enfermeiro: análise das publicações sobre o tema. Revi ciências medicas, v.15, n.3, p.241-248, 2006.

FLECK, Marcelo P. A et al. Aplicação da versão em português do instrumento de avaliação de qualidade de vida da
Organização Mundial da Saúde (WHOQOL-100). Rev. Saúde Pública [online], v.33, n.2, p.198-205, 1999.

KAWAKAME, Patrícia Moita Garcia; MIYADAHIRA, Ana Maria Kazue. Qualidade de vida de estudantes de graduação em enfermagem. Rev. esc. enferm. USP [online], v.39, n.2, p.164-172, 2005.

KIMURA, Miakoand; CARANDINA, Dirley Maria. Desenvolvimento e validação de uma versão reduzida do instrumento para avaliação da Qualidade de Vida no Trabalho de enfermeiros em hospitais. Rev. esc. enferm. USP [online], v.43, n.01, p.1044-1054, 2009.

MANETTI, et al. Revisando os fatores psicossociais do trabalho em enfermagem. Revi da Rede de Enfermagem do Nordeste, v.9, n.1, p.111-119, 2008.

MARTINS, Júlia Trevisan; ROBAZZI, Maria Lúcia do Carmo Cruz; BOBROFF, Maria Cristina Cescatto. Prazer e sofrimento no trabalho da equipe de enfermagem: reflexão à luz da psicodinâmica Dejouriana. Rev. esc. enferm. USP [online], v.44, n.4, p.1107-1111, 2008.

OLER, Fabiana G. et al. Qualidade de vida da equipe de enfermagem do centro cirúrgico. Rev. Arq Ciênc Saúde [online], v.12, n.2, p.102-110, 2005.

OLIVEIRA, Raquel Aparecida de; CIAMPONE, Maria Helena Trench. Qualidade de vida de estudantes de enfermagem: a construção de um processo e intervenções. Rev. esc. enferm. USP [online], v.42, n.1, p.57-65, 2008.

PIRES, et. al. Jornada de 30 horas semanais: condição necessária para assistência de enfermagem segura e de qualidade. Enfermagem em Foco, v.1, n.3, p.114-118, 2010.

RIOS, Kátia Assalvi; BARBOSA, Dulce Aparecida; BELASCO, Angélica Gonçalves Silva. Avaliação de qua- 
lidade de vida e depressão de técnicos e auxiliares de enfermagem. Rev. Latino-Am. Enfermagem [online]. v.18, n.3, p.413-420, 2010.

ROCHA, Sandra de Souza Lima; FELLI, Vanda Elisa Andres. Qualidade de vida no trabalho docente em enfermagem. Rev. Latino-Am. Enfermagem [online], v.12, n.1, p.28-35, 2004.

SANCHES, Viviane Francisco; CHRISTOVAM, Bárbara Pompeu; SILVINO, Zenith Rosa. Processo de trabalho do gerente de enfermagem em unidade hospitalar uma visão dos enfermeiros. Esc. Anna Nery [online], v.10, n.2, p.214-220, 2006.

SCHMIDT, Denise Rodrigues Costa; DANTAS, Rosana Aparecida Spadoti. Qualidade de vida no trabalho de profissionais de enfermagem, atuantes em unidades do bloco cirúrgico, sob a ótica da satisfação. Rev. Latino-Am. Enfermagem [online]., v.14, n.1, p.54-60, 2006.

SEIDL, Eliane Maria Fleury; ZANNON, Célia Maria Lana da Costa. Qualidade de vida e saúde: aspectos conceituais e metodológicos. Cad. Saúde Pública [online]., v.20, n.2, p.580-588, 2004.
SILVA, Amanda Aparecida; ROTENBERG, Lúcia; FISCHER, Frida Marina. Jornadas de trabalho na enfermagem: entre necessidades individuais e condições de trabalho. Rev. Saúde Pública [online]., v.45, n.6, p. 1117-1126. 2011.

SIQUEIRA JÚNIOR, Antônio Carlos; SIQUEIRA, Fernanda Paula Cerantola; GONÇALVES, Bárbara, Gisela de Oliveira Gôngora. $O$ trabalho noturno e a qualidade de vida dos profissionais de enfermagem. Rev. Min. Enferm [Internet], v.10, n.1, p.41-45, 2006.

SIQUEIRA, Vera Thania Alves; KURCGANT, Paulina. Satisfação no trabalho: indicador de qualidade no gerenciamento de recursos humanos em enfermagem, Rev Esc Enferm USP, v.46, n.1, p.146-152, 2014.

SOUZA, Ismelinda Maria Diniz Mendes et al. Qualidade de vida relacionada à saúde e sintomas depressivos de estudantes do curso de graduação em Enfermagem. Rev. Latino-Am. Enfermagem [online], v.20, n.4, p.736-743, 2012.
1. Enfermeira graduada pela UNIT - Aracaju / SE / Brasil, e-mail: rosianebsnane@hotmail.com

2. Enfermeira graduada pela UNIT - Aracaju / SE / Brasil, e-mail: carlascouto@hotmail.com

3. Mestre em saúde coletiva pela UEFS- Docente de Enfermagem - UNIT Aracaju / SE / Brasil, e-mail: ilva_ss@hotmail.com

4. Mestre em saúde e ambiente pela UNIT - Docente de Enfermagem - UNIT - Aracaju / SE / Brasil, e-mail: maria_pureza@unit.br 